\title{
Treatment of biliary stenosis using percutaneous transhepatic cholangiobiopsy with biopsy forceps of varying diameter
}

\author{
Zheng-Yang $\mathrm{Wu}^{1}$, De-Chao Jiao ${ }^{1}$, Fang-Fang Guo ${ }^{2}$, Dan-Dan Zhang ${ }^{3}$, Jian-Zhuang Ren ${ }^{1}$, Xin-Wei Han ${ }^{1}$ \\ ${ }^{1}$ Department of Interventional Radiology, the First Affiliated Hospital of Zhengzhou University, Zhengzhou, China; ${ }^{2}$ Department of Pathology, \\ Zhengzhou University People's Hospital \& Henan Provincial People's Hospital, Zhengzhou, China; ${ }^{3}$ Department of Pathology, the First Affiliated \\ Hospital of Zhengzhou University, Zhengzhou, China
}

Contributions: (I) Conception and design: ZY Wu, DC Jiao, XW Han; (II) Administrative support: JZ Ren, XW Han; (III) Provision of study materials or patients: ZY Wu, FF Guo, DD Zhang, DC Jiao; (IV) Collection and assembly of data: ZY Wu, FF Guo, DD Zhang; (V) Data analysis and interpretation: ZY Wu, FF Guo, DD Zhang, DC Jiao; (VI) Manuscript writing: All authors; (VII) Final approval of manuscript: All authors.

Correspondence to: Xin-Wei Han. Department of Interventional Radiology, The First Affiliated Hospital of Zhengzhou University, No. 1 Jianshe East Road, Erqi District, Zhengzhou 450052, China. Email: fcchanxw@zzu.edu.cn.

Background: The present study aimed to compare the clinical results and pathological diagnostic quality of percutaneous transhepatic cholangiobiopsy for biliary obstruction using biopsy forceps (BFs) of varying diameter.

Methods: A total of 57 patients with obstructive jaundice who underwent percutaneous transhepatic cholangiobiopsy and drainage with 1 of 2 BFs diameters (6.0-mm BFs, n=30; 4.5-mm BFs, n=27) between February 2018 and May 2019 were retrospectively assessed. BFs were compared in terms of their sample quality, diagnostic accuracy, sensitivity, specificity, number of passes, and complication rate.

Results: All 57 patients underwent the procedure successfully and the technical success rate was $100 \%$. The 6.0- and 4.5-mm BFs demonstrated a diagnostic accuracy of 80\% (24/30) and 85\% (23/27), respectively $(\mathrm{P}=0.733)$, and a sensitivity of $78 \%(22 / 28)$ and $86 \%(22 / 26)$, respectively $(\mathrm{P}=0.729)$. The specificity of both the 6.0 - and $4.5-\mathrm{mm}$ BFs was $100 \%$. The complication rate was $10 \%(3 / 30)$ with the $6.0-\mathrm{mm}$ BFs and $19 \%$ (5/27) with the $4.5-\mathrm{mm}$ BFs $(\mathrm{P}=0.456)$. The mean number of biopsies was $2.9 \pm 0.6$ with the $6.0-\mathrm{mm}$ BFs compared with 3.6 \pm 1.0 with the $4.5-\mathrm{mm}$ BFs $(\mathrm{P}<0.001)$. The $6.0-\mathrm{mm}$ BFs provided a larger biopsy size and a less crushed specimen compared with the 4.5 -mm BFs. The overall tissue scores were $5.2 \pm 0.8$ with 6.0 -mm BFs and $4.5 \pm 1.0$ with $4.5-\mathrm{mm} \mathrm{BFs}(\mathrm{P}=0.012)$.

Conclusions: There was no statistically significant difference in the clinical results between the 2 BFs in the context of percutaneous transhepatic cholangiobiopsy. Superior samples were obtained using the $6.0-\mathrm{mm}$ $\mathrm{BFs}$, with a fewer number of passes. The complication rate did not increase compared with the 4.5-mm BFs.

Keywords: Percutaneous transhepatic cholangiobiopsy; obstructive jaundice; biliary obstruction; biopsy forceps

Submitted Feb 08, 2021. Accepted for publication May 28, 2021.

doi: $10.21037 /$ qims-21-161

View this article at: https://dx.doi.org/10.21037/qims-21-161

\section{Introduction}

Obstructive jaundice is a common consequence of biliary stenosis, the reason of biliary stenosis has a complex pathogenesis. Laboratory evidence and imaging are inadequate to determine whether biliary stenosis is benign or malignant; thus, subsequent treatment is often unguided. Early lesions are small, and it is difficult to conduct histopathological examinations using direct percutaneous puncture.

Percutaneous transhepatic cholangiobiopsy (PTCB) 
Table 1 Patient characteristics and overall data

\begin{tabular}{lccc}
\hline Characteristics & $6.0-\mathrm{mm} \mathrm{BFs}(\mathrm{n}=30)$ & $4.5-\mathrm{mm}$ BFs $(\mathrm{n}=27)$ & $\mathrm{P}$ value \\
\hline Age (years) & $64.6 \pm 9.7$ & $64.1 \pm 9.4$ & 0.896 \\
Male & $14(47 \%)$ & $14(52 \%)$ & 0.695 \\
TBIL (mmol/L) & $207.4 \pm 47.4$ & $222.5 \pm 65.0$ & 0.317 \\
ALT $(\mathrm{U} / \mathrm{L})$ & $123.9 \pm 42.0$ & $126.6 \pm 48.6$ & 0.818 \\
CA199 level $>40 \mathrm{U} / \mathrm{mL}$ & 17 & 15 & 0.932 \\
Site of the lesion & & & 0.848 \\
Hilar & 5 & 4 & 0.582 \\
CHD & 8 & 9 & 0.975 \\
CBD & 9 & 6 & 0.697 \\
Periampullary segment & 8 & 8 &
\end{tabular}

BFs, biopsy forceps; TBIL, total bilirubin; ALT, alanine aminotransferase; CHD, common hepatic duct; CBD, common bile duct.

is simple to perform, minimally invasive, and is deemed an accurate and reliable new method for bile duct histopathological diagnosis in clinical practice (1-3). A series of studies have compared biopsy forceps (BFs) in the context of digestive tract endoscopy. It is now accepted that larger cups provide larger biopsies (4-6). However, the biliary duct is different from the digestive tract. Larger cups require a larger transhepatic tract, which may increase the complication rate. It is currently not certain how small BFs influence complication rates and sample quality, and. to the best of our knowledge, no studies have investigated this issue. In this study, we aimed to compare clinical and pathological results using 2 different sizes of BFs in the context of biliary stenosis.

\section{Methods}

\section{Patients}

The study was conducted in accordance with the tenets of the Declaration of Helsinki (as revised in 2013) and was approved by the ethics committee of our hospital. All patients provided written informed consent for the interventional procedure. Patients referred for percutaneous transhepatic cholangiodrainage (PTCD) to treat obstructive jaundice between February 2018 and May 2019 were retrospectively reviewed. Suspected malignant obstruction was the main indication for biopsy. A total of 57 patients were included in the study; patient characteristics are shown in Table 1. Every patient underwent computed tomography (CT) or magnetic resonance imaging (MRI) prior to biopsy, and then underwent PTCB with either a $6.0-\mathrm{mm}(\mathrm{n}=30)$ or $4.5-\mathrm{mm}(\mathrm{n}=27)$ BFs being used. All procedures were performed by 2 experienced interventional radiologists with at least 5 years of clinical experience. All biopsy specimens were examined using histology techniques, and the results were evaluated by an experienced pathologist.

All patients received a pathological diagnosis obtained through PTCB. When pathological and clinical diagnoses were consistent, the pathological findings were considered positive. If no tumor was identified by pathological analysis but imaging and clinical evidence supported a tumor diagnosis, the pathological findings were considered negative. Each patient's diagnosis was determined by 2 senior interventional radiologists (RJZ and $\mathrm{XWH}$ ) with 25 and 35 years of experience, respectively, via consultation of imaging, laboratory, prognostic, and follow-up data.

\section{Biopsy forceps}

The 2 BFs were manufactured by Micro-Tech (Nanjing, China). Both BFs are disposable. The BFs comprise a fenestrated cup without a needle and an oval cup with a smooth edge. All BFs had sheath-coated shafts designed for smooth insertion. The $2 \mathrm{BFs}$ differed in their caliper diameter $(6.0 v s .4 .5 \mathrm{~mm})$, and were designed for $2.8-\mathrm{mm}$ and 2.0-mm channels, respectively (Figure 1).

\section{Percutaneous transhepatic cholangiobiopsy technique}

Under local anesthesia, РTCB was conducted using 


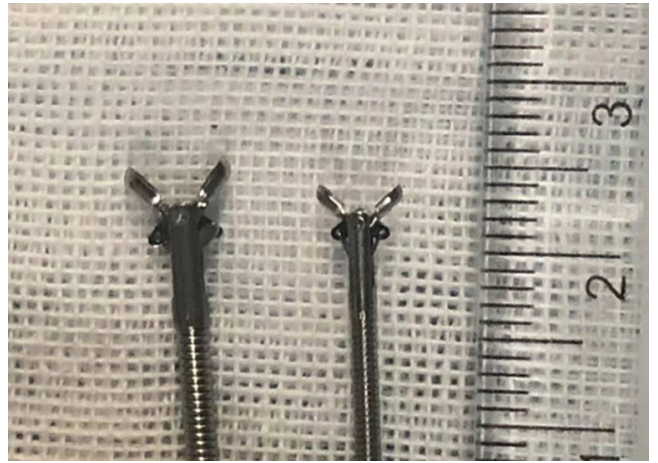

Figure 1 The 6.0-mm biopsy forceps (left) and 4.5-biopsy forceps (right) with open cup.

a 21-G Chiba needle punctured into the biliary tract percutaneously. After the structure of the biliary tree was visualized, a 0.014-inch platinum guide wire (Cook Medical, Bloomington, IN, USA) was introduced into the intrahepatic bile duct through the trocar sheath of a Chiba needle (Cook Medical). As the guide wire was kept in place, the trocar sheath was withdrawn. A 5-F 3-piece dilator (Cook Medical) was then introduced to dilate the pathway while the stylet of the dilator and the guide wire were withdrawn, keeping the sheath of the dilator in place. The sheath was extracted after insertion of a 0.035 inch guide wire (Terumo, Tokyo, Japan) to the upper end of the stenosis through the sheath. A 5-F KMP catheter (Cook Medical) was advanced along the guide wire, and the catheter and guide wire were passed through the stenosis simultaneously into the duodenum or jejunum (Figure 2A).

For the $4.5-\mathrm{mm} \mathrm{BFs,} \mathrm{a} 7-\mathrm{F}(23 \mathrm{~cm})$ catheter sheath (Cordis, Hialeah, FL, USA) and a 0.018-in guide wire (Terumo) were used. For the $6.0-\mathrm{mm} \mathrm{BFs}$, a 0.035 -in guide wire and a 9-F long sheath were used. The sheath was then advanced over the wire up to the proximal limit of the stricture. The BFs were then passed through the sheath alongside the guide wire, and the cup was opened and pushed forward. It was then closed and retracted to remove the tissue specimen (Figure $2 B$ ). The procedure was repeated to obtain as much tissue as possible. Generally, 2 rice-sized tissue samples were obtained from each patient. On some occasions, 3 or more PTCB procedures were conducted as necessary. The number of biopsies was recorded for each patient.

Subsequent biliary interventional treatment was conducted after PTCB. A biliary drain was inserted depending on the type of stricture, and a stent was considered when the pathological result was malignant (Figure 2C). Patients were monitored for 24 hours postoperatively to observe any decline in blood pressure or possible complications such as hemobilia, bile leakage, or hematemesis.

\section{Histological assessment}

A histological examination was performed by a specialist pathologist who was blinded to the BFs type used to obtain specimens. Each piece of tissue on each slide was evaluated in terms of its depth, size, artifact, and fixation. However, due to the design of the study, the fixation score was not considered in the overall tissue score. The score of the 3 remaining parameters defined the overall tissue score, which aimed to assess the global quality of each biopsy $(7,8)$.

For all parameters, a score of 0 (inadequate), 1 (questionable), or 2 (adequate) was attributed to each piece of tissue. In regard to depth, the tissue was qualified as adequate if sufficient mucosa and muscularis were included. The depth was designated as questionable if sufficient mucosa without muscularis was included. The piece of tissue was considered inadequate in terms of its depth if no mucosa was included. The pathologist assigned a size score depending on whether the tissue size was subjectively considered as inadequate, questionable, or adequate. Artifact scores were given based on crushing and splitting according to the following criteria: $0 \geq 75 \%$ crushing or splitting; $1=$ between $25 \%$ and $75 \%$ crushing or splitting; and $2 \leq 25 \%$ crushing or splitting.

\section{Statistical analysis}

All statistical analyses were performed using SAS 9.13 (SAS Institute, Cary, NC, USA). Continuous variables are reported as mean \pm standard deviation, while categorical variables are reported as proportions. To compare categorical variables, the chi-square test or Fisher's exact test were used. To compare continuous data, an independent samples $t$ test was performed. A $\mathrm{P}$ value of $<0.05$ was considered statistically significant.

\section{Results}

All 57 patients underwent PTCB with a technical success rate of $100 \%$. No severe adverse events (AEs) were observed according to The Society of Interventional Radiology (SIR) Scale (9). The mean number of biopsies using the $6.0-\mathrm{mm}$ 

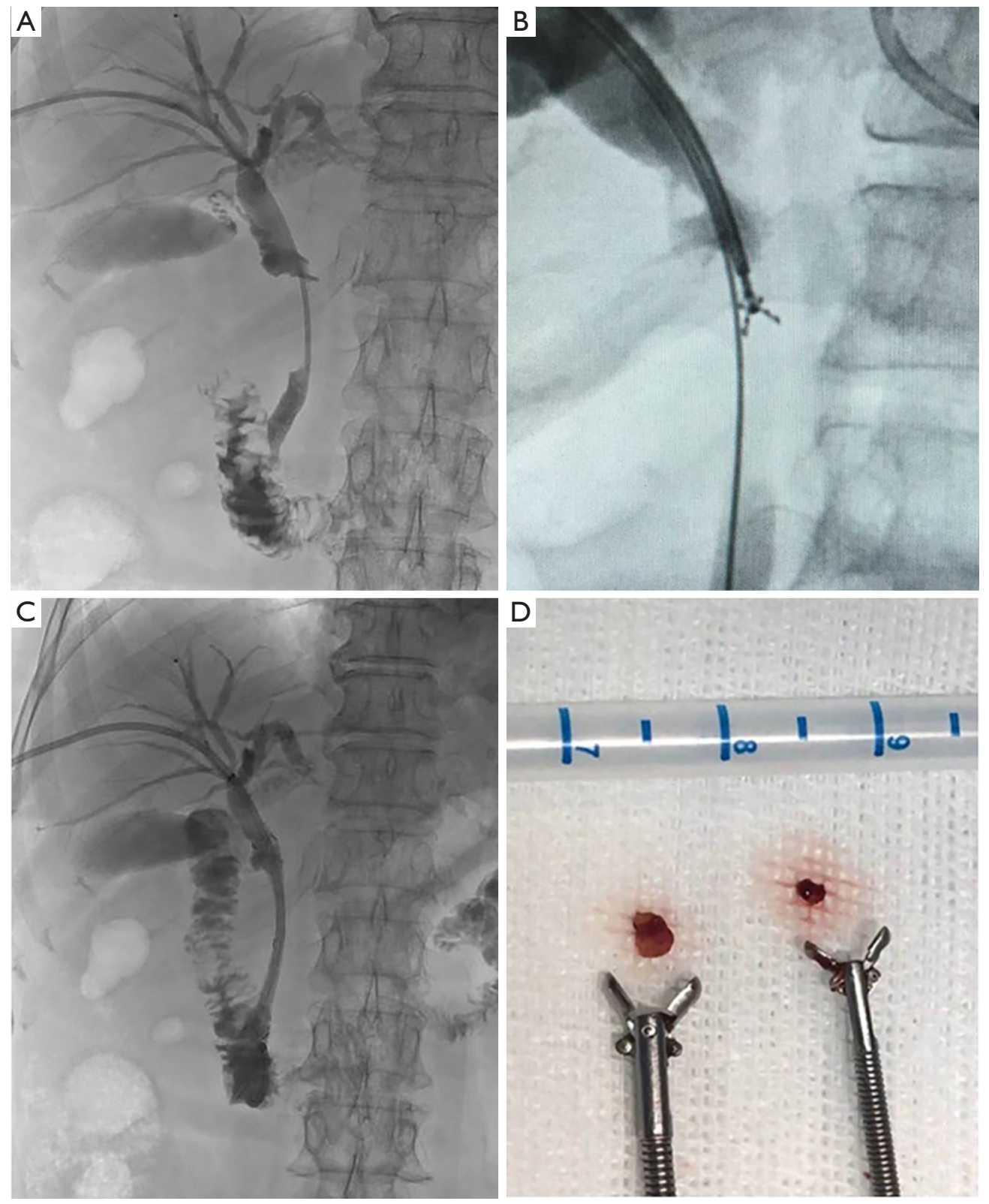

Figure 2 (A) Percutaneous transhepatic cholangiography (PTC) shows an occlusion in the common bile duct; (B) PTCB was performed; (C) a drainage tube and stent were implanted; (D) tissues were obtained using 6.0- and 4.5-mm biopsy forceps.

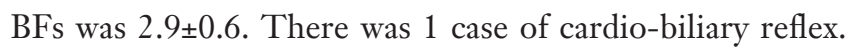
This patient complained of chest pain during the procedure. He also experienced bradycardia with a heart rate of 45 beats per minute, which was treated successfully with atropine. Additionally, there were 2 cases of hemobilia. The minor complications rate was $10 \%(3 / 30)$. In all, 28 patients were clinically diagnosed with a malignant biliary stricture, and 2 patients were clinically diagnosed with benign biliary stricture; 24 patients received a positive pathological diagnosis, with 22 cases being malignant, and 2 cases being benign. The overall diagnostic accuracy and sensitivity were $80 \%(24 / 30)$ and $78 \%(22 / 28)$, respectively. There were no false-positive results, and the specificity was $100 \%$.

A total of 27 patients underwent the procedure with the 4.5 -mm BFs. The mean number of biopsies was 3.6 1.0 , which was significantly different when compared with the 
Table 2 Results of percutaneous transhepatic cholangiobiopsy in 57 cases

\begin{tabular}{|c|c|c|c|c|c|c|}
\hline & \multicolumn{3}{|c|}{ 6.0-mm BFs (pathological result) } & \multicolumn{3}{|c|}{ 4.5-mm BFs (pathological result) } \\
\hline Hilar cholangiocarcinoma & 6 & 6 & 0 & 8 & 8 & 0 \\
\hline Pancreatic carcinoma & 4 & 3 & 1 & 2 & 2 & 0 \\
\hline Metastasis & 2 & 0 & 2 & 2 & 0 & 2 \\
\hline Biliary tract inflammation & 1 & 0 & 1 & 1 & 0 & 1 \\
\hline
\end{tabular}

BFs, biopsy forceps.

Table 3 Diagnostic yield using the 6.0 - and 4.5 -mm BFs with histology

\begin{tabular}{lccc}
\hline & $6.0-\mathrm{mm} \mathrm{BFs}, \mathrm{n}=30$ & $4.5-\mathrm{mm}$ BFs, $\mathrm{n}=27$ & 0.538 \\
\hline True positive (TP) & 22 & 22 & 1.000 \\
True negative (TN) & 2 & 1 & 0.733 \\
False negative (FN) & 6 & 4 & - \\
False positive (FP) & 0 & 0 & 0.733 \\
Diagnostic, \% & & & 0.729 \\
Accuracy & 80 & 85 & - \\
Sensitivity & 78 & 86 & 100 \\
Specificity & 100 & 19 & 0.456 \\
Complication rate & 10 & &
\end{tabular}

Accuracy $=(T P+T N) / N$; sensitivity $=T P /(T P+F N)$; specificity $=T N /(T N+F P)$.

mean number of biopsies performed using the $6.0-\mathrm{mm}$ BFs $(\mathrm{P}=0.001)$. There was 1 case of bile leak on cholangiography and 4 cases of hemobilia, which spontaneously resolved within 48 hours. The complication rate was 19\% (5/27). A total of 26 patients were clinically diagnosed as having malignant lesions. Furthermore, 1 patient was diagnosed with benign biliary stricture; 23 patients received a positive pathological diagnosis with 22 being malignant lesions and 1 being a benign lesion. The diagnostic accuracy and sensitivity were $85 \%(23 / 27)$ and $86 \%(22 / 26)$, respectively, with a specificity of $100 \%$. There were no differences in accuracy, sensitivity, specificity, or complication rate between the 2 BFs types $(\mathrm{P}>0.05)$. The results of clinical and pathological diagnosis are shown in Tables 2,3.

The histological assessment scores are listed in Table 4. Larger tissue samples could be obtained using the $6.0-\mathrm{mm}$ BFs with a 1-time biopsy (Figure 2D). The size of the specimen acquired with the $6.0-\mathrm{mm}$ BFs was greater than that with the 4.5 -mm BFs $(1.8 \pm 0.4 v s .1 .5 \pm 0.5$, respectively; $\mathrm{P}=0.025)$. In obtaining sufficient specimen, the number of passes required with the $4.5-\mathrm{mm}$ BFs was significantly greater than that required with the $6.0-\mathrm{mm} \mathrm{BFs}(\mathrm{P}=0.001)$. A greater number of passes with the $4.5-\mathrm{mm}$ BFs could reach the same depth as that reached by the $6.0-\mathrm{mm}$ BFs $(1.7 \pm 0.4$ vs. $1.7 \pm 0.5 ; \mathrm{P}=0.807)$, but this might have resulted in a greater degree of crushing and splitting (Figure 3). 
Table 4 Histological score for depth, size, and artifacts in biliary tissue specimens

\begin{tabular}{|c|c|c|c|c|c|c|c|c|c|}
\hline & \multicolumn{3}{|c|}{ Depth } & \multicolumn{3}{|c|}{ Size } & \multicolumn{3}{|c|}{ Artifacts } \\
\hline 6.0-mm BFs & 0 & 8 & 22 & 0 & 7 & 23 & 0 & 8 & 22 \\
\hline 4.5-mm BFs & 0 & 8 & 19 & 0 & 14 & 13 & 1 & 15 & 11 \\
\hline$P$ & \multicolumn{3}{|c|}{0.807} & \multicolumn{3}{|c|}{0.025} & \multicolumn{3}{|c|}{0.009} \\
\hline
\end{tabular}

BFs, biopsy forceps.
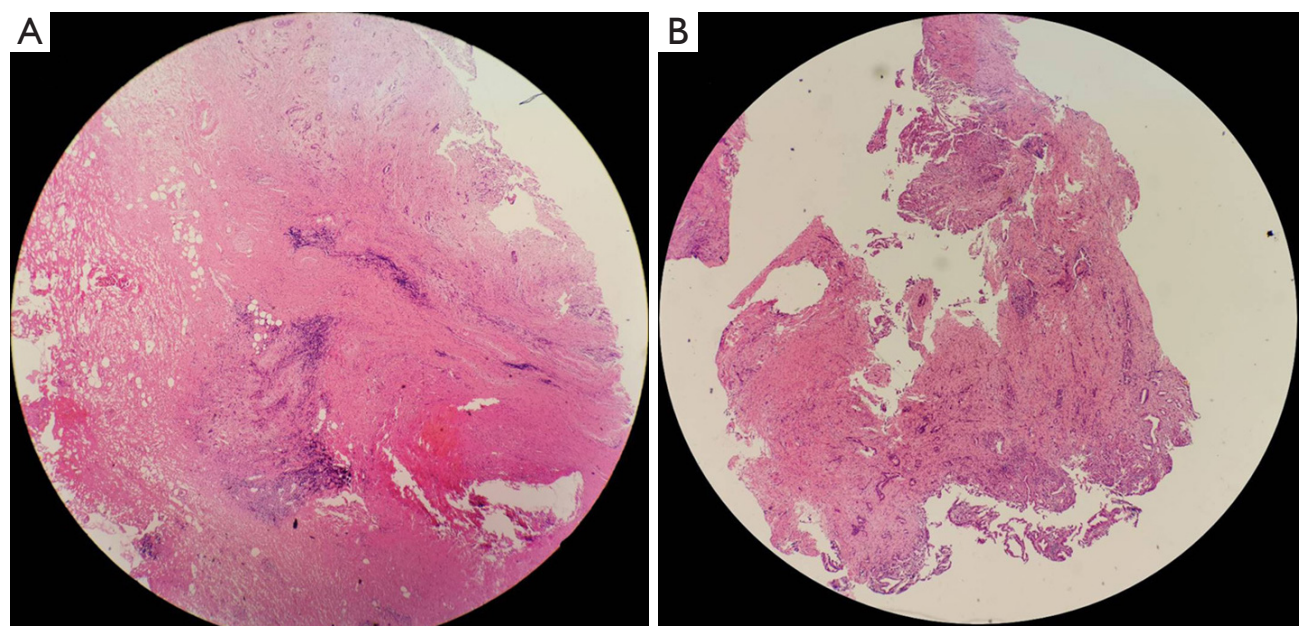

Figure 3 Pathology image of cholangiocarcinoma (hematoxylin and eosin, $\times 40$ ). The size and artifacts of the specimen obtained using the 6.0-mm biopsy forceps (A) were superior to those obtained using 4.5-mm biopsy forceps (B).

There were fewer artifacts with the $6.0-\mathrm{mm}$ BFs compared with the $4.5-\mathrm{mm}$ BFs $(1.7 \pm 0.4$ vs. $1.4 \pm 0.6$, respectively; $\mathrm{P}=0.009)$. Hence, the overall tissue score obtained by the $6.0-\mathrm{mm}$ BFs was $5.2 \pm 0.8$, which was greater than that obtained with the 4.5-mm BFs $(4.5 \pm 1.0 ; \mathrm{P}=0.012)$.

\section{Discussion}

The site of bile duct obstruction can be identified quickly and accurately using noninvasive imaging systems, such as ultrasound, CT, or MRI. However, tumors that originate from the biliary epithelium are often too small to have a specific image appearance. A diagnosis of the cause of biliary obstruction is essential to providing appropriate treatment to patients presenting with obstructive jaundice. PTCD is a well-established interventional radiologic procedure that is used when patients are deemed unsuitable for surgical resection. A diagnostic sample performed during PTCD may be essential for management of these patients (10-12).

PTCB has become a popular method for diagnosing biliary tumors since it was first reported in 1980 (13). Currently, various transluminal techniques for acquiring tissue from biliary tumors are performed through a percutaneous transhepatic tract (1-3,10-13). Because most bile duct tumors arise from the ductal epithelium, tissue obtained from an abnormal segment of the bile duct seems to be most appropriate for pathological examination. Histological diagnoses with BFs are more successful than are diagnoses made by assessing bile cytology (sensitivity $44-67 \%$ ) or fine-needle aspiration biopsies (sensitivity $45 \%)$, with a reported sensitivity of $71-93 \%(10,11,14,15)$.

In this study, we retrospectively reviewed and analyzed data from patients who underwent percutaneous transhepatic cholangiobiopsy and drainage. The results show that forceps biopsy with $6.0-\mathrm{mm}$ and $4.5-\mathrm{mm}$ BFs is safe and effective for tissue sampling of the bile tract. The technical success rate was $100 \%$. With 6.0 - and 4.5-mm BFs, the diagnostic accuracy and sensitivity were $80 \%(24 / 30)$ and $85 \%(23 / 27 ; \mathrm{P}=0.733)$, and $78 \%(22 / 28)$ and $86 \%(22 / 26 ; \mathrm{P}=0.729)$, respectively. The specificity of 
both BFs was $100 \%$, which is in accordance with the results of previous studies $(10,11,14-16)$.

For improved diagnostic accuracy and clinical efficiency, larger specimens are preferred by pathologists. We noticed that larger tissue samples could be obtained using the $6.0-\mathrm{mm}$ BFs with a 1 -time procedure. The $6.0-\mathrm{mm} \mathrm{BFs}$ could satisfy this requirement with a fewer number of passes compared with the $4.5-\mathrm{mm}$ BFs. For the same amount of sample, a greater number of passes were needed with the $4.5-\mathrm{mm}$ BFs. This may lead to a greater number of artifacts, which result in a lower overall tissue score and may influence the pathological assessment. Furthermore, excessive force in biopsy resulted in 1 case of bile leakage, which involved 7 passes with the $4.5-\mathrm{mm}$ BFs. Cardio-biliary reflex and hemobilia are often reported in patients who have only a PTC, and hence, we believe these complications are not due solely to PTCB. The complications associated with PTCB seem to relate to the number of passes rather than the size of the BFs. There were slightly more complications with the $4.5-\mathrm{mm}$ BFs $(5 / 27,19 \%)$ than with the $6.0-\mathrm{mm}$ BFs $(3 / 30,10 \%)$, although this difference was not statistically significant.

The present study has several limitations. First, the sample size was relatively small, which reduced the statistical power of the analysis. However, some results did achieve statistical significance. Second, using 2 BFs on a single patient may lead to more convincing outcomes; however, considering the cost of the instruments and the surgical duration, this was not adopted. Additionally, the forceps were selected randomly, and this might have led to selection bias. Finally, it is possible that a lesion may be malignant with benign-appearing imaging and a false negative biopsy. False-negative results might have gone unrecognized due to this. Nevertheless, there was no malignant clinical or imaging evidence of such lesions during follow up.

\section{Conclusions}

We found no statistically significant difference in the clinical results obtained from the 2 BFs in the context of percutaneous transhepatic cholangiobiopsy. The quality of samples obtained using the $6.0-\mathrm{mm}$ BFs was superior, and fewer passes were required. The complication rate did not differ significantly between the $6.0-\mathrm{mm}$ BFs and $4.5-\mathrm{mm}$ BFs.

\section{Acknowledgments}

Funding: None.

\section{Footnote}

Conflicts of Interest: All authors have completed the ICMJE uniform disclosure form (available at https://dx.doi. org/10.21037/qims-21-161). The authors have no conflicts of interest to declare.

Ethical Statement: The authors are accountable for all aspects of the work in ensuring that questions related to the accuracy or integrity of any part of the work are appropriately investigated and resolved. The study was conducted in accordance with the tenets of the Declaration of Helsinki (as revised in 2013) and was approved by the ethics committee of our hospital. All patients provided written informed consent for the interventional procedure.

Open Access Statement: This is an Open Access article distributed in accordance with the Creative Commons Attribution-NonCommercial-NoDerivs 4.0 International License (CC BY-NC-ND 4.0), which permits the noncommercial replication and distribution of the article with the strict proviso that no changes or edits are made and the original work is properly cited (including links to both the formal publication through the relevant DOI and the license). See: https://creativecommons.org/licenses/by-nc-nd/4.0/.

\section{References}

1. Li Z, Li TF, Ren JZ, Li WC, Ren JL, Shui SF, Han XW. Value of percutaneous transhepatic cholangiobiopsy for pathologic diagnosis of obstructive jaundice: analysis of 826 cases. Acta Radiol 2017;58:3-9.

2. Warnken EM, Uder M, Stein H, Wucherer M, Lell M, Muschweck H, Adamus R. Transhepatic forceps biopsy after PTCD for histological assessment of bile duct stenoses or occlusions. Z Gastroenterol 2019;57:133-8.

3. Park JG, Jung GS, Yun JH, Yun BC, Lee SU, Han BH, Ko $\mathrm{JH}$. Percutaneous transluminal forceps biopsy in patients suspected of having malignant biliary obstruction: factors influencing the outcomes of 271 patients. Eur Radiol 2017;27:4291-7.

4. Bernstein DE, Barkin JS, Reiner DK, Lubin J, Phillips RS, Grauer L. Standard biopsy forceps versus large-capacity forceps with and without needle. Gastrointest Endosc 1995;41:573-6.

5. Danesh BJ, Burke M, Newman J, Aylott A, Whitfield P, Cotton PB. Comparison of weight, depth, and diagnostic adequacy of specimens obtained with 16 different biopsy 
forceps designed for upper gastrointestinal endoscopy. Gut 1985;26:227-31.

6. Elmunzer BJ, Higgins PD, Kwon YM, Golembeski C, Greenson JK, Korsnes SJ, Elta GH. Jumbo forceps are superior to standard large-capacity forceps in obtaining diagnostically adequate inflammatory bowel disease surveillance biopsy specimens. Gastrointest Endosc 2008;68:273-8; quiz 334, 336.

7. Willard MD, Lovering SL, Cohen ND, Weeks BR. Quality of tissue specimens obtained endoscopically from the duodenum of dogs and cats. J Am Vet Med Assoc 2001;219:474-9.

8. Dahan J, Semin MO, Monton C, Amiriantz S, Concordet D, Raymond-Letron I, Dossin O. Comparison of routinely used intestinal biopsy forceps in dogs: an ex vivo histopathological approach. J Small Anim Pract 2017;58:162-7.

9. Omid Khalilzadeh, Mark O Baerlocher, Paul B Shyn, Bairbre L Connolly, A Michael Devane, Christopher S Morris, Alan M Cohen, Mehran Midia, Raymond H Thornton, Kathleen Gross, Drew M Caplin, Gunjan Aeron, Sanjay Misra, Nilesh H Patel, T Gregory Walker, Gloria Martinez-Salazar, James E Silberzweig, Boris Nikolic. Proposal of a New Adverse Event Classification by the Society of Interventional Radiology Standards of Practice Committee. J Vasc Interv Radiol 2017;28:14321437.e3.

10. Tapping CR, Byass OR, Cast JE. Cytological sampling versus forceps biopsy during percutaneous transhepatic

Cite this article as: $\mathrm{Wu} \mathrm{ZY}$, Jiao DC, Guo FF, Zhang DD, Ren JZ, Han XW. Treatment of biliary stenosis using percutaneous transhepatic cholangiobiopsy with biopsy forceps of varying diameter. Quant Imaging Med Surg 2022;12(1):207214. doi: $10.21037 /$ qims-21-161 biliary drainage and analysis of factors predicting success. Cardiovasc Intervent Radiol 2012;35:883-9.

11. Patel P, Rangarajan B, Mangat K. Improved Accuracy of Percutaneous Biopsy Using "Cross and Push" Technique for Patients Suspected with Malignant Biliary Strictures. Cardiovasc Intervent Radiol 2015;38:1005-10.

12. Tibana TK, Grubert RM, Fornazari VAV, Barbosa FCP, Bacelar B, Oliveira AF, Marchiori E, Nunes TF. The role of percutaneous transhepatic biliary biopsy in the diagnosis of patients with obstructive jaundice: an initial experience. Radiol Bras 2019;52:222-8.

13. Elyaderani MK, Gabriele OF. Brush and forceps biopsy of biliary ducts via percutaneous transhepatic catheterization. Radiology 1980;135:777-8.

14. Jung GS, Huh JD, Lee SU, Han BH, Chang HK, Cho YD. Bile duct: analysis of percutaneous transluminal forceps biopsy in 130 patients suspected of having malignant biliary obstruction. Radiology 2002;224:725-30.

15. Ierardi AM, Mangini M, Fontana F, Floridi C, De Marchi G, Petrillo M, Capasso R, Chini C, Cocozza E, Cuffari S, Segato S, Rotondo A, Carrafiello G. Usefulness and safety of biliary percutaneous transluminal forceps biopsy (PTFB): our experience. Minim Invasive Ther Allied Technol 2014;23:96-101.

16. Li TF, Ren KW, Han XW, Li WC, Ren JL, Jiao DC, Li Z, Ma J. Percutaneous transhepatic cholangiobiopsy to determine the pathological cause of anastomotic stenosis after cholangiojejunostomy for malignant obstructive jaundice. Clin Radiol 2014;69:13-7. 